\title{
REMARKS ON THE WAVE FRONT OF A DISTRIBUTION $\left({ }^{1}\right)$
}

\author{
BY
}

\section{AKIVA GABOR}

ABSTRACT. Basic facts about composition and multiplication of distributions as given in [1] are proved using the formulas for the wave front set of the image and pullback of distributions.

1. Let $X$ be a manifold, $D^{\prime}(X)$ the distributions on $X$. If a smooth density, $d x$, is given on $X$, then any smooth function on $X, g$, gives rise to a smooth distribution

$$
\langle f, g\rangle=\int f g d x .
$$

These smooth distributions can be pulled back along differentiable maps $\phi$ : $Y \rightarrow X$ (that is, $g \in D^{\prime}(X)$ goes to $\phi^{*} g \in D^{\prime}(Y)$ by the usual pullback of functions). We would like to define a "continuous" extension of this and other notions, so as to include a wider subset of $D^{\prime}(X)$. This was done in [1, Chapter 2.5], and here we only give a unified treatment with some slight extensions.

According to [1] the question of when a distribution $A$ can be pulled back is answered in terms of a set, $W F(A) \subset T^{*}(X)$, and a formula for $W F\left(\phi^{*} A\right)$ is obtained when $\phi^{*} A$ is defined. If $\phi: X \rightarrow Z$ is a differentiable map and $A$ satisfies obvious conditions on its support relative to $A$ then $\phi_{*} A$ can be defined as a distribution on $Z$. We derive a formula relating $W F\left(\phi_{*} A\right)$ to $W F(A)$. The various theorems of [1] on multiplication and composition of distribution then follow from standard functional constructions.

Definition. If $A \in D^{\prime}(X),\left(x_{0}, k_{0}\right) \in T^{*}(X), k_{0} \neq 0$, then $\left(x_{0}, k_{0}\right) \notin W F(A)$ (wave front of $A$ ) if there exists a $C^{\infty}$ function $\phi$ with compact support, $\phi\left(x_{0}\right) \neq$ 0 , and an open cone $\Gamma \subset T_{x_{0}}^{*}(X)$ containing $k_{0}$, such that for each $k \in \Gamma$ and some $C^{\infty}$ function $\psi$ with $d \psi\left(x_{0}\right)=k$,

$$
t^{m}\left|A\left(\phi e^{-i t \psi}\right)\right| \rightarrow 0 \quad(t \rightarrow \infty) .
$$

This definition is geometric ( $w$ ith no reference to local coordinates in $X$ ), and is

Received by the editors August 10, 1971.

AMS 1969 subject classifications. Primary 4640; Secondary 3520.

Key words and phrases. Distribution, wave front set.

(1) The se results were obtained during participation in a joint seminar held by the mathematics department of the Weizmann Institute and the physics department of the University of Tel-Aviv. One of the topics of the seminar was [1]. I would like to thank Professors B. Kostant, B. Simon and S. Sternberg for helpful comments. 
easily seen to be equivalent to the one given in [1] via Proposition 2.5.5 and the independence of coordinates given by Theorem 2.5.11' thereof.

In terms of local coordinates, let $U \subset X, x_{0} \in U$, be identified with an open subset of $V=\mathbf{R}^{n}$. Then the tangent space at each point of $U$ is identified with $V$, and the cotangent space with $V^{*}$, so that the cotangent bundle is $U \times V^{*}$. For a distribution $u$ on $X$, with compact support in $U$, we have the Fourier transform, $\hat{u}$, given by

$$
\hat{u}(k)=\left\langle e^{-i(k, x)}, u\right\rangle \quad \text { for } k \in V^{*} \text {. }
$$

With this notation, $\left(x_{0}, k_{0}\right) \notin W F(A)$ for $A$ as above if there is $\phi \in C_{0}^{\infty}, \phi\left(x_{0}\right) \neq 0$, an open cone $\Gamma \subset V^{*}$ and a constant $C_{m}$ for each $m$, such that

$$
|\widehat{\phi A}(k)| \leq C_{m}(1+|k|)^{-m} \text { for all } k \in \Gamma,|k| \geq C \text {. }
$$

For distributions with support in $U$ we define convergence as follows:

If $\Gamma$ is a closed cone in $T^{*}(U)$, define

$$
D_{\Gamma}^{\prime}(u)=\left\{A \in D^{\prime}(U): W F(A) \subset \Gamma\right\}
$$

A sequence $A_{j} \in D_{\Gamma}^{\prime}(U)$ converges to $A \in D_{\Gamma}^{\prime}(U)$ if

(i) $A_{j} \rightarrow A$ in $D^{\prime}(U)$ (weakly),

(ii) $|k|^{m}\left|\hat{A}_{j}(k)-\hat{A}(k)\right| \rightarrow 0,|k| \rightarrow \infty$, uniformly for $k$ in a closed cone disjoint of $\Gamma$.

For a general manifold $X$ we define convergence in $D_{\Gamma}^{\prime}(X)$ by partition of unity. A definition utilizing pseudodifferential operators can be found in [1] together with a proof that $C^{\infty}(X)$ is sequentially dense in $D_{\Gamma}^{\prime}(X)$.

2. The following theorem is proved directly in [1, Theorem 2.5.11']:

Theorem 1. Let $X, Y$ be manifolds, $\phi: Y \rightarrow X$ a $C^{\infty}$ map, and denote the set of normals of the map-

$$
N_{\phi}=\left\{(\phi(y), \xi) \in T^{*}(X):\langle\eta, \xi\rangle=0 \text { for all } \eta \in \phi_{*} T_{y}\right\} .
$$

If $A \in D^{\prime}(X)$ and $W F(A) \cap N_{\phi}=\varnothing$, we can define the pullback $\phi^{*} A$ in one and only one way so that it is equal to the composition $f \circ \phi$ when $A=f$ smooth, and is sequentially continuous from $D_{\Gamma}^{\prime}(X)$ to $D^{\prime}(Y)$ for any closed cone $\Gamma \subset T^{*}(X) \backslash 0$ with $\Gamma \cap N_{\phi}=\not \varnothing$. Moreover,

$$
W F\left(\phi^{*} A\right) \subset \phi^{*} W F(A)=\left\{\left(y, \phi_{y}^{*}(\xi)\right):(\phi(y), \xi) \in W F(A)\right\} .
$$

Let $\phi: X \rightarrow Y$ be a $C^{\infty}$ map, $A \in D^{\prime}(X)$, and suppose that either

(a) $\phi$ is proper $\left(\phi^{-1}(K)\right.$ compact for any $K \subset Y$ compact $)$ or

(b) $A$ has compact support. 
Then $\phi_{*} A$ is well defined as a distribution on $Y$ by the formula

$$
\left\langle f, \phi_{*} A\right\rangle=\left\langle\phi^{*} f, A\right\rangle \text {. }
$$

For any $S \subset T^{*}(X)$ we define $\phi_{*} S \subset T^{*}(Y)$ by

$$
\phi_{*} S=\bigcup_{x}\left(\phi_{x}^{*}\right)^{-1}\left(S_{x}\right) \text { where } S_{x}=S \cap T_{x}^{*}(X)
$$

If $S$ is compact, so is $\phi_{*} S$.

Also, given a pair of mappings $\phi_{1}: X \rightarrow Y, \phi_{2}: Y \rightarrow \sim Z$,

$$
\left(\phi_{2} \circ \phi_{1}\right)(s)=\phi_{1 *}\left(\phi_{2 *} s\right) \text {. }
$$

Theorem 2. $W F\left(\phi_{*} A\right) \subset \phi_{*} W F(A)$ for $A \in D(X)$ as above.

Proof. Every map can be factored as $\phi=\pi \circ \iota$, where $\iota: X \rightarrow X \times Y$ is $x \mapsto(x, \phi(x))$, and $\pi: X \times Y \rightarrow Y$ is projection onto the second factor. It therefore suffices to prove the theorem for the cases where $\phi=\imath$ is an imbedding and $\phi=\pi$ is projection as above.

In the case that $\iota: X \rightarrow Y$ is an imbedding, we can make the more refined assertion that

$$
W F\left(\iota_{*} A\right)=\iota_{*} W F(A) .
$$

The $W F$ is independent of coordinates, and is defined locally so we can apply a partition of unity and change of coordinates. Therefore, it suffices to prove this in the case where $X \subset \mathbf{R}^{m}, Y=X \times Z, Z \subset \mathbf{R}^{n}$, and ८ defined by $\iota(x)=(x, 0)$. In this case, for any $S \subset T^{*}(X)$,

$$
\iota_{*}(S)=S \times T_{0}^{*}(Z), \quad T^{*}(X \times Z)=T^{*}(X) \times T^{*}(Z), \quad \iota_{*}(A)=A \otimes \delta .
$$

This gives immediately $W F\left(\iota_{*} A\right)=\iota_{*} W F(A)$ by the next (independent) theorem on the wave front of a tensor-product (for $C$ ) and direct observation (for $\supset$ ).

Now we examine the case $\phi=\pi: X \times Y \rightarrow Y$. Here $\phi_{x}^{*}(l)=(0, l)_{(x, y)}$ for any $l \in T_{y^{*}}^{*}$ Thus $l \in \phi_{*}(S)$ iff $\exists x$ with $(0, l)_{(x, y)} \in S$. Suppose that $l_{0} \notin \phi_{*}(W F(A))$. Then for each $x,\left(0, l_{0}\right)_{(x, y)} \notin W F(A)$. By assumption the possible $(x, y) \in \phi^{-1}(y)$ form a compact set. Each $(x, y)$ has a coordinate neighborhood and $\psi$ such that $\left\langle e^{-i(l . y)}, \psi A\right\rangle$ vanishes infinitely rapidly at infinity for all $l$ in a conical neighborhood of $l_{0}$. By compactness, a finite number of cones will do. By applying a partition of unity w.r. to $x$ we are reduced to the case where $A$ is of compact support, and the result follows from the definition.

Theorem 3. If $A \in D^{\prime}(X), B \in D^{\prime}(Y)$ with $\Gamma_{1}=W F(A) \subset T^{*}(X), \Gamma_{2}=W F(B)$ $\subset T^{*}(Y)$, then

$$
W F(A \otimes B) \subset \Gamma_{1} \times \Gamma_{2} \cup \Gamma_{1} \times 0_{y} \cup 0_{x} \times \Gamma_{2}
$$


(where $0_{x}=\{(x, 0)\} \subset T^{*}(X)$ the zero section).

The proof is direct from the definitions.

Note that these three operations $\left(\phi^{*}, \phi_{*}\right.$ and $\left.\otimes\right)$, as well as compositions of them, are sequentially continuous. This will be a useful tool in extending other operations in a sequentially continuous mode.

3.

Theorem [1, Theorem 2.5.10]. Let $\Gamma_{1}, \Gamma_{2}$ be two closed cones in $T^{*}(X) \backslash 0$ satisfying $\Gamma_{1}+\Gamma_{2} \subset T^{*}(X) \backslash 0$ (where $\left.\Gamma_{1}+\Gamma_{2}=\left\{\left(x, \xi_{1}+\xi_{2}\right):\left(x, \xi_{j}\right) \in \Gamma_{j}\right\}\right)$. Then the product $A_{1} A_{2}$ of $A_{j} \in D_{j}^{\prime}(X)$ can be defined (in a unique way) and

$$
W F\left(A_{1} A_{2}\right) \subset \Gamma_{1}+\Gamma_{2} \cup \Gamma_{1} \cup \Gamma_{2} .
$$

Proof. For functions $f_{1}, f_{2} \in D(X)$ the product is

$$
f_{1}(x) \cdot f_{2}(x)=\Delta^{*}\left(f_{1} \otimes f_{2}\right)
$$

where $f_{1} \otimes f_{2}$ is in $D(X \times X), f_{1} \otimes f_{2}(x, y)=f_{1}(x) f_{2}(y)$, and $\Delta: X \rightarrow X \times X$ is the diagonal map $\Delta: x \rightarrow(x, x)$ with $\Delta^{*} g(x)=g(x, x)$ for any $g(x, y) \in D(X, Y)$.

In view of our previous remark, a multiplication in $D^{\prime}(X)$ must also satisfy $A_{1} A_{2}=\Delta *\left(A_{1} \otimes A_{2}\right)$ whenever it is defined.

The only restriction on the above construction is on applying $\Delta^{*}$.

For any distribution $A \in D^{\prime}(X \times X)$ and the diagonal map $\Delta$ the restriction is $W F(A) \cap N_{\Delta}=\varnothing$ where $N_{\Delta}=\left\{(x, x, \eta,-\eta): \eta \in T^{*}(X)\right\}$.

In our case, $A_{j} \in D_{\Gamma_{j}}^{\prime}(X)$, so

$$
W F\left(A_{1} \otimes A_{2}\right) \subset \Gamma_{1} \times \Gamma_{2} \cup \Gamma_{1} \times 0_{x} \cup 0_{x} \times \Gamma_{2}
$$

and $W F\left(A_{1} \otimes A_{2}\right) \cap N_{\Delta}=\varnothing$ means exactly $\Gamma_{1}+\Gamma_{2} \subset T^{*}(X) \backslash 0$ as we required. So $A_{1} A_{2}$ is defined, and

$$
W F\left(A_{1} A_{2}\right) \subset \Delta^{*}\left(\Gamma_{1} \times \Gamma_{2} \cup \Gamma_{1} \times 0_{x} \cup 0_{x} \times \Gamma_{2}\right)=\Gamma_{1}+\Gamma_{2} \cup \Gamma_{1} \cup \Gamma_{2} . \quad \text { Q.E.D. }
$$

Let $K \in D^{\prime}(X, Y)$. It defines a continuous map $K: C_{0}^{\infty}(Y) \rightarrow D^{\prime}(X)$ by

$$
\langle\psi, K \phi\rangle=K(\psi \otimes \phi) \quad \text { for } \phi \in C_{0}^{\infty}(Y), \psi \in C_{0}^{\infty}(X)
$$

Theorem [1, Theorem 2.5.12]. For any $u \in C_{0}^{\infty}(Y), W F(K u) \subset \pi_{*}(W F(K))$ (where $\pi: X \times Y \rightarrow X) . \pi_{*} W F(K)$ is also denoted $W F_{x}(K)=\{(x, \xi):(x, \xi, y, 0) \epsilon$ $W F(K)$ for some $y \in Y\}$.

Proof. $u$ gives $1 \otimes u \in D^{\prime}(X \times Y)$, which is smooth. Therefore the product $K(1 \otimes u)$ is defined and $K u=\pi_{*} K(1 \otimes u)$.

$$
W F(K(1 \otimes u)) \subset W F(K)+W F(1 \otimes u) \cup W F(K) \cup W F(1 \otimes u)
$$


but $1 \otimes u$ is smooth, so $W F(K(1 \otimes u)) \subset W F(K)$ which gives $W F(K u) \subset \pi_{*} W F(K)$.

Theorem [1, Theorem 2.5.14]. Let $K \in D^{\prime}(X \times Y)$, and denote by

$$
W F^{\prime}(K)=\left\{(x, \xi, y,-\eta) \in T^{*}(X) \times T^{*}(Y):(x, \xi, y, \eta) \in W F(K)\right\}
$$

a relation mapping sets in $T^{*}(Y) \backslash 0$ to sets in $T^{*}(X) \backslash 0$. If $W F_{x}(K)=\varnothing, u \epsilon$ $D^{\prime}(Y)$ and $W F(u) \cap W F_{Y}^{\prime}(K)=\varnothing$ (where $W F_{Y}^{\prime}(K)=\{(y, \eta):(x, 0, y,-\eta) \in W F(K)$ for some $x\}$ ) then

$$
W F(K u) \subset W F^{\prime}(K) W F(u)
$$

for $K u \in D^{\prime}(X)$ defined by

$$
\langle\phi, K u\rangle=\langle\phi \otimes 1, K(1 \otimes u)\rangle, \quad \phi \in C_{0}^{\infty}(x) .
$$

Proof. If everything is defined,

$$
K u=\pi_{*}(K(1 \otimes u)), \quad \pi: X \times Y \rightarrow X .
$$

Since $W F_{x}(K)=\varnothing, K(1 \otimes u)$ is defined and

$$
W F(K(1 \otimes u)) \subset W F(K)+0_{x} \times W F(u) \cup W F(K) \cup 0_{x} \times W F(u) .
$$

For any $P, Q \subset T^{*}(X), \pi_{*}(P \cup Q)=\pi_{*}(P) \cup \pi_{*}(Q)$. By our assumption $\pi_{*} W F(K)=$ $\varnothing$, and $\pi_{*}\left(0_{x} \times W F(u)\right)=\varnothing$ always, so $W F(K u) \subset \pi_{*}\left(W F(K)+0_{x} \times W F(u)\right)=$ $W F^{\prime}(K) W F(u)$. Q.E.D.

Let $K_{1} \in D^{\prime}(X \times Y), K_{2} \in D^{\prime}(Y \times Z)$ be properly supported (that is, $\pi_{*} K_{j}$ are well defined for the projections $\pi$ on the components). Denote $\Gamma_{j}=W F\left(K_{j}\right)$.

Theorem [1, Theorem 2.5.15]. If $\Gamma_{1} \times 0_{z}+0_{x} \times \Gamma_{2} \subset T^{*}(X \times Y \times Z) \backslash 0$, then the composition $K=K_{1} \circ K_{2} \in D^{\prime}(X \times Z)$ is well defined, and

$$
W F^{\prime}\left(K_{1} \circ K_{2}\right) \subset W F^{\prime}\left(K_{1}\right) \circ W F^{\prime}\left(K_{2}\right) \cup\left(W F_{x}\left(K_{1}\right) \times 0_{z}\right) \cup\left(0_{x} \times W F_{z}^{\prime}\left(K_{2}\right)\right) .
$$

Proof. For smooth distributions of the form

$$
K_{1}=f(x) \otimes g_{1}(y), \quad K_{2}=g_{2}(y) \otimes b(z),
$$

the composition is defined as $f(x) \otimes b(z) \cdot\left\langle g_{1}, g_{2}\right\rangle$. In other words,

$$
\left(f \otimes g_{1}\right) \circ\left(g_{2} \otimes b\right)=\pi_{*}\left(\left(f \otimes g_{1} \otimes 1\right)\left(1 \otimes g_{2} \otimes b\right)\right)
$$

where $\pi: X \times Y \times Z \rightarrow X \times Z$.

In the continuous extension of this definition, we define, where possible, $K_{1} \circ K_{2}=\pi_{*}\left(\left(K_{1} \otimes 1_{z}\right)\left(1_{x} \otimes K_{2}\right)\right)$ for the above $\pi$.

The restriction on defining products limits us to the case $0 \notin \Gamma_{1} \times 0_{z}+0_{x} \times$ $\Gamma_{2}$. This holding, we get

$$
\begin{aligned}
& W F\left(K_{1} \circ K_{2}\right) \subset \pi_{*}\left(\Gamma_{1} \times 0_{z}+0_{x} \times \Gamma_{2} \cup \Gamma_{1} \times 0_{z} \cup 0_{x} \times \Gamma_{2}\right) \\
& =W F^{\prime}\left(K_{1}\right) \circ W F\left(K_{2}\right) \cup\left(W F_{x}\left(K_{1}\right) \times 0_{z}\right) \cup\left(0_{x} \times W F_{z}\left(K_{2}\right)\right)
\end{aligned}
$$


or

$$
W F^{\prime}\left(K_{1} \circ K_{2}\right) \subset W F^{\prime}\left(K_{1}\right) \circ W F^{\prime}\left(K_{2}\right) \cup\left(W F_{x}\left(K_{1}\right) \times 0_{z}\right) \cup\left(0_{x} \times W F_{z}^{\prime}\left(K_{2}\right)\right) . \quad \text { Q.E.D. }
$$

Note that $0 \notin \Gamma_{1} \times 0_{z}+0_{x} \times \Gamma_{2}$ holds in particular when $W F_{Y}^{\prime}\left(K_{1}\right) \cap W F_{Y}\left(K_{2}\right)$ $=\varnothing$, which is the restriction in [1], however composition exists in less restrictive circumstances.

\section{REFERENCE}

1. L. Hörmander, Fourier integral operators. I, Acta Math. 127 (1971), 79-183. DEPARTMENT OF MATHEMATICS, WEIZMANN INSTITUTE OF SCIENCE, REHOVOT, ISRAEL 